\title{
An Adaptive Gray Scale Watermarking Method in Wavelet Domain
}

\author{
Jialing Han $^{1,2}$ Xiaohui Zhao ${ }^{2}$ \\ ${ }^{1}$ School of Management Science and Information Engineering, \\ Jilin University of Finance and Economics, Changchun, P.R. China \\ ${ }^{2}$ College of Communication Engineering, Jilin University, \\ Changchun, P.R. China \\ weiya2000@163.com
}

\begin{abstract}
In the past a few years, many watermarking approaches have been proposed for solving the copyright protection problems. This paper presents a new gray scale watermarking method in wavelet domain based on the compression of the watermark and the pretreatment of the original image. Through the compression of watermark, it can greatly reduce the embedding capacity of the watermark, which can embed more watermark bits in the host image. Through the preprocessing of the original image based on human visual system's characteristic, it can ensure the imperceptibility. The use of threshold $T$ can adaptively determine the quantity of embedded blocks, and control the capacity of embedded secret information. The experimental results show that our method has an excellent transparency and robustness.
\end{abstract}

Keywords: Watermark compression, image preprocessing, discrete wavelet transform

\section{Introduction}

With the emergence of internet and multimedia technology, the security issue of digital transmission and the copyright protection of digital products have attracted more and more people's attention. How to protect the security of digital products better in the current network environment has attracted widespread attention. Therewith, an emerging concept of information hiding, the digital watermark technology, has been put forward. The digital watermark technology is a branch of information hiding, and its basic idea is embedding the secret information into the digital video, image or audio, which is aimed to protect the copyright of digital products. The secret information can be the unique serial number to identify the user, and it can also be related information of products as the authentication of products to deal with the dispute, and this secret information is the digital watermark. The digital watermark is divided into image watermark, audio watermark, video watermark and the like according to different formats.

Depending on the work domain that a watermark is embedded in, watermarking techniques can be divided into two main categories: spatial domain [1-6] and transform domain. Watermarking in spatial domain means modifying the spatial characteristics directly, such as pixel values and statistical traits. Such methods are convenient and fast, but vulnerable to attacks. In contrast, transform domain algorithms first convert the media into transform domains.

Such as discrete cosine transform, discrete Fourier transform, discrete wavelet transform, singular value decomposition, etc. Then, a watermark is embedded by modifying the frequency coefficients. Because of the consistency with current compression standard, discrete cosine transform and discrete wavelet transform methods attract many researchers' attention [7-12]. 
Hsieh and Tseng [13] proposed a watermark embedding method based on qualified significant wavelet tree. This method is deduced on a basis of the zero-tree wavelet algorithm. The watermark information is embedded into two sub-bands of every wavelet tree.

Lin [14] proposed a quantization watermarking algorithm based on significant difference of pieces. Every seven wavelet coefficient of sub-band are divided into a group to form a block, and embed the watermark through tuning the difference value between two maximal wavelet coefficients of the block. The total capacity of embedded watermark information depends on the size of blocks.

Mainly, there are three aspects to be noticed in watermarking design, imperceptibility, capacity and robustness. Imperceptibility means the watermark should be perceptually invisible, the difference between the original and output image should be as small as possible. Capacity means the quantity of information to be embedded, which is expected to be as large as possible. Robustness means that the watermark must be difficult to remove. Commonly, these aspects restrict each other. When we want to improve robustness of the watermarked image, we should increase the watermarking intensity, but at the same time imperceptibility of the image have depressed. When we want to increase the capacity, the imperceptibility must be reduced. On the contrary, decreasing watermarking intensity when we intend to enhance imperceptibility, robustness is depressed.

At present, most of the watermarking algorithms take use of binary watermark images, but the use of gray scale watermarks is rarely. Compared with the binary watermarks, gray watermark images can contain more visual information and content, so hiding information is more difficult. In this paper, we propose a new gray scale watermarking method in wavelet domain based on the compression pretreatment of the watermark and the pretreatment of the original image, it can greatly reduce the embedding bits of the watermark, or we can say it can embed more watermark bits in the host image.

In the rest of this paper, we explain the details of proposed scheme and its performance as follows. In Section 2, the proposed watermark preprocessing scheme is explained in detail. The digital watermark algorithm is described in Section 3. The simulation results and analysis are presented in Section 4. Finally, conclusion is given in Section 5.

\section{Watermark Preprocessing}

The traditional digital watermark technologies usually use 0-1 bits as watermark information. Now with the rapid development of multimedia technology and Internet, people like to use images to express and convey information more and more. Compared with text and audio information, image information has a more intuitive and easier property to be accepted by people. Now there are a lot of watermarking algorithms using image watermark. However, most algorithms take use of binary watermark images. Compared with the binary watermarks, gray watermark images can contain more visual information and content. The research of this paper is based on the gray-scale watermark. But the corresponding is that compared with the binary watermark, gray watermark image will occupy more storage space, and the bandwidth of transmission will consume more resources. Therefore, in order to better use of gray image as secret information and reduce storage space, this paper puts forward using image compression encoding technology which compresses gray-scale watermark image firstly.

In recent years, people have made some progress in the field of image compression encoding. Predictive coding technology is one of the important branch. This technology constructs a forecasting encoder based on image pixel correlation, to eliminate the redundancy between pixels to achieve the purpose of compression.

The principle of predictive coding technology is as follows. For a particular sequence $\left\{x_{n} \mid n=1,2, \ldots\right\}$, according to the condition entropy theory, we can estimate the 
value of $x_{n}$ from the known $x_{n-1}, x_{n-2}, \cdots, x_{n-p}$, the predicted value is $x_{n}^{\prime}$, the predicted error is $e_{n}$.

$$
\begin{gathered}
x_{n}^{\prime}=\sum_{i=1}^{p} a_{i} x_{n-p} \\
e_{n}=x_{n}-x_{n}^{\prime}
\end{gathered}
$$

The number of bits needed to encode prediction error is far less than the number of bits needed to encode the original data. Thus, we can achieve the goal of the compressed data. For gray image, the image pixel is denoted by gray value, and there is inevitable correlation between adjacent pixels. So adjacent pixels can be completely used to forecast, and then to compress.

Assuming that the original watermark image is $s(m, n)$, the pixels in a two-dimensional linear prediction is

$$
s^{\prime}(m, n)=\sum_{(k, l) \in S} \sum a_{k l} s(m-k, n-l)
$$

Where, $a_{k l}$ is the two-dimensional forecast coefficient, $(k, l) \in S$ represent forecasting by using points in the $S$ field. The commonly used two-dimensional linear prediction formula is as follows:

$$
s^{\prime}(m, n)=a_{10} s(m-1, n)+a_{01} s(m, n-1)+a_{11} s(m-1, n-1)
$$

By this way, we can embed the predicted error $e$ as the secret information into the original image, the capacity of $e$ is much less than the original watermark. That is to say, we can embed large capacity of secret images, for whatever large the primary watermark image. In here, we embed the linear prediction coefficients instead of embedding the pixel values.

\section{The Digital Watermark Method}

\subsection{Image Preprocessing}

Every host image has its own information and texture features, and every image often contains complex and smooth regions. Most of the past watermarking schemes do not take this into account, all pixels or blocks have the same chances to join the embedding process.

Based on human visual system's characteristic, edges and complex texture of image have good visual mask effect. If the secret information is embedded into these regions, it means more imperceptibility.

In our study, for the purpose of getting complex regions in host image and taking less time, the host image is first divided into non-overlapped sub-blocks, and then the blockvariance method is employed, which computes each sub-block's variance for detecting complex regions.

In our experiments, a block size is chosen to be $8 \times 8$. The block classification is described as follows. Divide the original cover image into non-overlapped sub-blocks $u \times v$ in spatial domain, each block is represented as $B_{k}, \mathrm{k}$ is the index of the block.

$$
\begin{gathered}
m_{k}=\frac{1}{u \times v} \sum_{i=1}^{u} \sum_{j=1}^{v} B_{k}(i, j) \\
v_{k}=\frac{1}{u \times v} \sum_{i=1}^{u} \sum_{j=1}^{v}\left(B_{k}(i, j)-m_{k}\right)^{2}
\end{gathered}
$$


Where $m_{k}$ is the mean value of block $B_{k}$, and $v_{k}$ represents the variance of block $B_{k}$. Variance can measure the relative smoothness and contrast of the intensity in a region.

For each block $B_{k}$, we sort out the variance $v_{k}$ and then compute the image's median variance. To represent the complex of each block, a block mark map $M_{k}$ is used and defined as

$$
M_{k}= \begin{cases}0 . & \text { if } v_{k} \geq T \\ 1 . & \text { otherwise }\end{cases}
$$

$\mathrm{T}$ is a threshold to determine the smoothness or complex of image, and whether to be embedded or not. The value of $\mathrm{T}$ can use to control the quantity of embedded blocks, and can also control the capacity of embedded secret information. While, the value of $\mathrm{T}$ is determined by $v_{k}$, which mean the variance of block. In this paper, we use mean values of all $v_{k}$. The following Figure 1 show the experimental results when the value of $\mathrm{T}$ is determined. In the figures, the showed blocks are complex blocks, which are selected to be embedded, and the black parts are not.

If we want to embed more secret information in the host image, we should add more available embedded position, what needs to reduce the threshold value of T. For example, when the value of $\mathrm{T}$ is $1 / 2$ of the mean value, the quantity of chosen blocks will increase, and the overall complexity may reduce relative to the value of $\mathrm{T}$. As a result, although larger number of secret information can be embedded, the concealment of the image will drop.

On the other hand, to increase masking and visibility, it can be done by raising the threshold $\mathrm{T}$, such as making $\mathrm{T}$ take 2 times of the mean value. In this way, the number of selected blocks for embedding secret information will decrease. As a result, the embedded secret information will decline. That is to say, adding masking is at the expense of reducing the embedding capacity.

The two are a pair of irreconcilable contradiction. Therefore, it can be chose according to the specific need of users. The results of 'Lena', 'Baboon' and 'Barbara' image after texture region analysis using our algorithm with different $\mathrm{T}$ are shown in Figure 2 and Table 1.
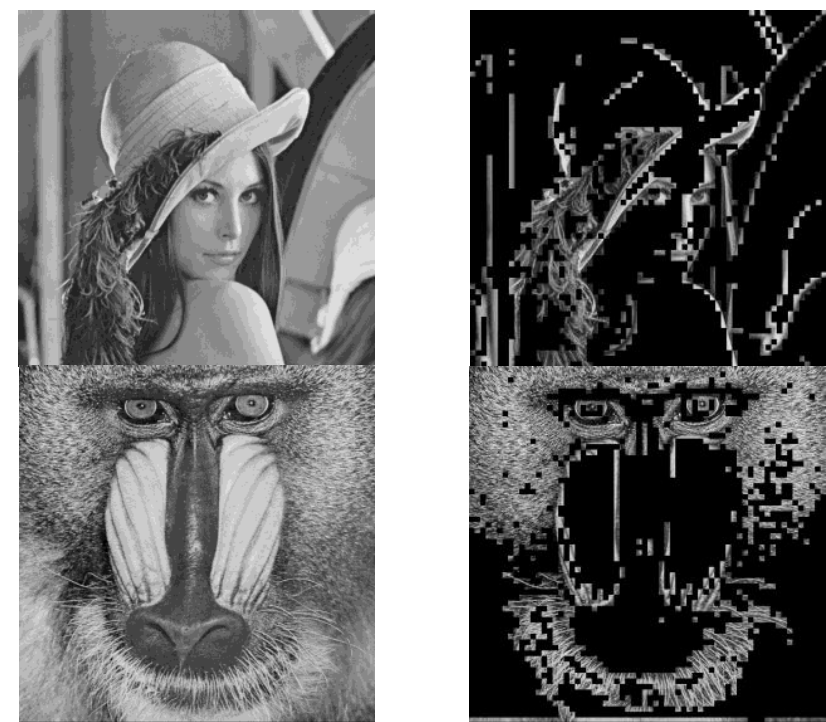

Figure 1. Result Images of Lena and Baboon Texture Region Analysis Using Block Variance Method 


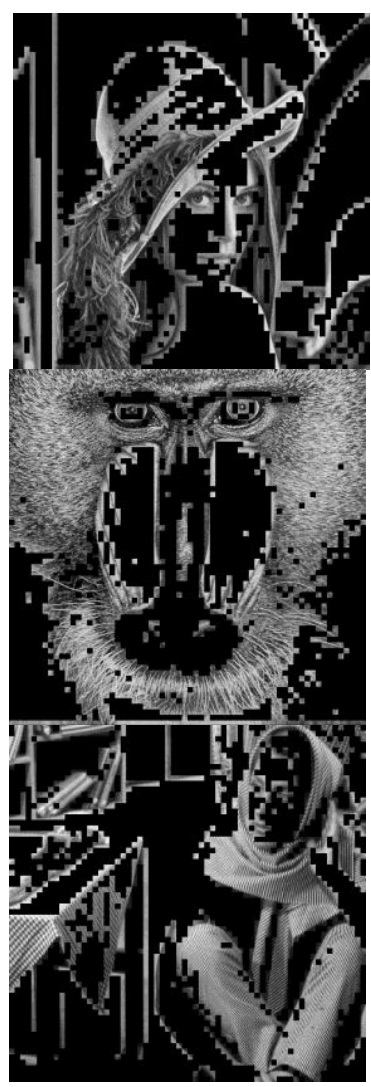

$\mathrm{T}=\mathrm{mean} / 2$

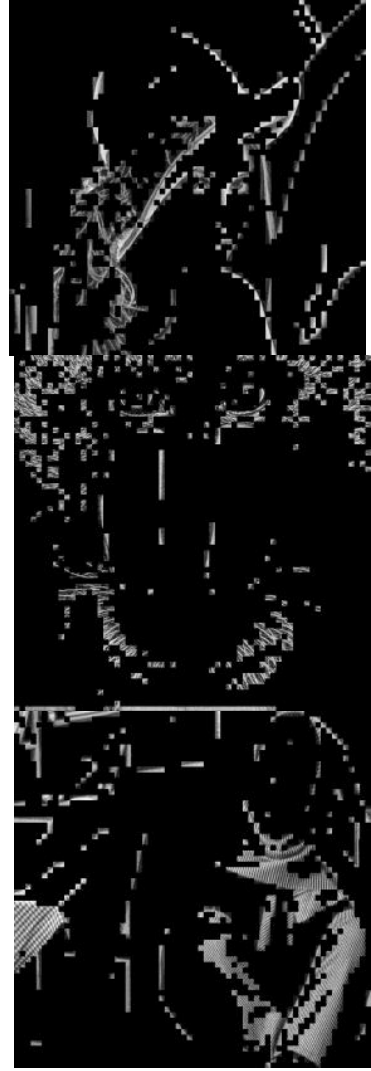

$\mathrm{T}=2 *$ mean

Figure 2. Results of Lena, Baboon and Barbara image after texture region analysis with different $T$

Table 1. Capacity with Different T

\begin{tabular}{|c|c|c|}
\hline Image & The value of T & $\begin{array}{c}\text { The quantity of } \\
\text { satisfied blocks }\end{array}$ \\
\hline \multirow{3}{*}{ Lena } & 129.7870 & 1303 \\
& 259.5741 & 957 \\
& 519.1482 & 590 \\
\hline \multirow{3}{*}{ Baboon } & 431.6616 & 2509 \\
& 863.3232 & 1782 \\
& 1726.6464 & 556 \\
\hline \multirow{3}{*}{ Barbara } & 249.5923 & 1872 \\
& 499.1845 & 1389 \\
& 998.3690 & 765 \\
\hline
\end{tabular}

\subsection{Watermark Embedding}

Comparing with the arithmetic of the spatial domain, the frequency domain arithmetic has some more advantages, like stronger robustness, etc. In here, we choose to embed in the wavelet domain.

- Step 1: Transform the texture active region sub-images using discrete wavelet transform. 
- Step 2: Insert the linear prediction coefficients $e$ as secret information.

$$
\text { - } \quad L w^{\prime}=L w(1+e \beta)
$$

Here, $L w$ expresses the original wavelet coefficients, $\beta$ expresses the embedding strength, and $L w^{\prime}$ expresses the wavelet coefficients after embedding.

- Step 3: Perform the inverse discrete wavelet transform on $L w^{\prime}$. Embed the watermarked sub-images back into the original host image to get the watermarked image.

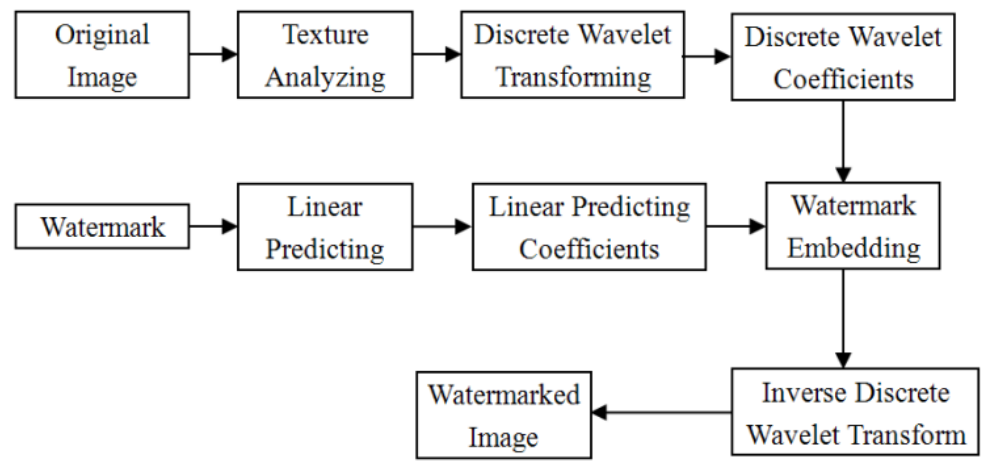

Figure 3. The Block-diagram of Embedding Algorithm

\subsection{Watermark Extracting}

First use the pretreatment method above to find texture active region by the threshold T. Then perform the discrete wavelet transform on these sub-images. Watermarking extraction algorithm is the inverse method of the embedding algorithm. The watermark can be extracted just when we get embedding strength $\beta$ as the secret keys. The prediction coefficients are obtained as follows.

$$
e=\left(L w^{\prime}-L w\right) /(\beta L w)
$$

Using the prediction coefficients $e$ and each pixel value of its neighbors, we can get the watermark information.

\section{Experimental Results and Analysis}

This chapter first describes the running environment of the experiment. All algorithms are simulated under the software MATLAB R2009a. We use 8 gray-level test images of size $512 \times 512$ obtained from USC-SIPI image database for our experiments, namely Lena, Baboon, Barbara, Pepper, Boat, Elaine, Lake and Airplane. These images were used to determine how the difference images influences the performance of the proposed method.
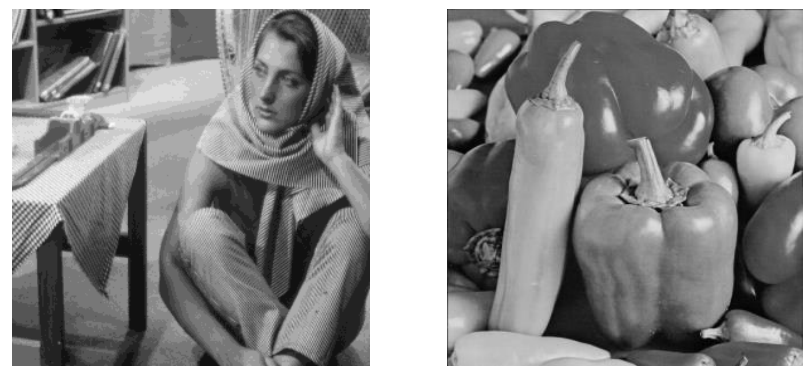

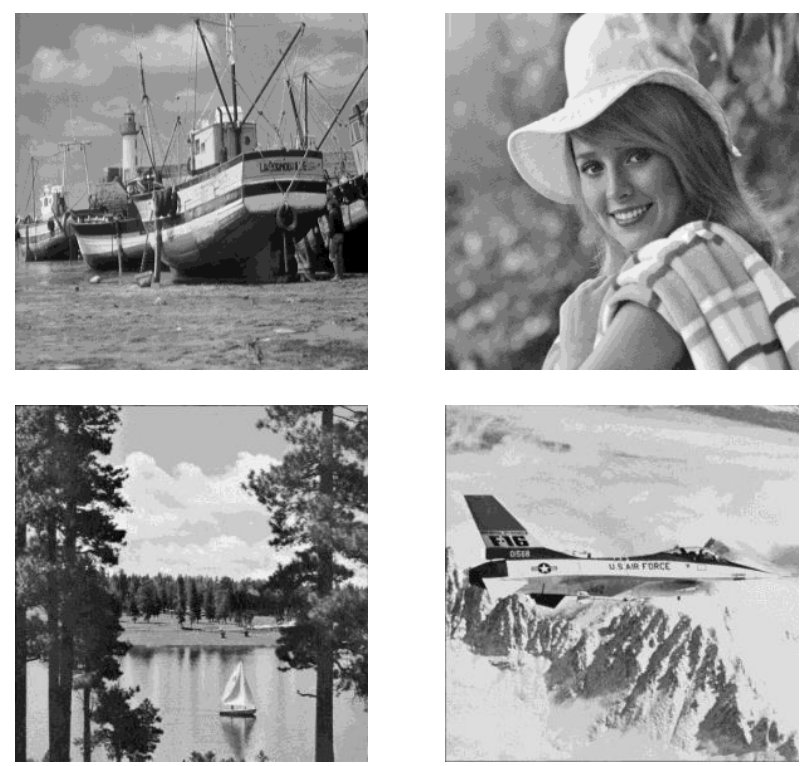

\section{Figure 4. Other Test Images: Barbara, Pepper, Boat, Elaine, Lake and Airplane}

The measurement of the general watermarking system consists of two aspects: the invisibility and robust. Invisibility can be calculated by peak signal to noise ratio (PSNR) to evaluate the quality between the watermarked image and the original image. The PSNR is formulated as follow:

$$
\begin{gathered}
P S N R=10 \times \log _{10}\left(\frac{\max \left(I^{2}(i, j)\right)}{M S E}\right)(d B) \\
M S E=\frac{1}{M \times N} \sum_{i=1}^{M} \sum_{j=1}^{N}\left[I(i, j)-I^{\prime}(i, j)\right]^{2}
\end{gathered}
$$

MSE is the mean-square error between the host image and the watermarked image. M and $\mathrm{N}$ are the height and width of the image respectively. $I(i, j)$ and $I^{\prime}(i, j)$ are the values of the coordinate $(i, j)$ in the original image and the watermarked image respectively. Higher PSNR value indicates better invisibility is achieved. When the value of PSNR is more than $30 \mathrm{~dB}$, the human eye cannot detect the difference between the host image and the watermarked image. The PSNRs of the 8 images are shown in Figure 5.

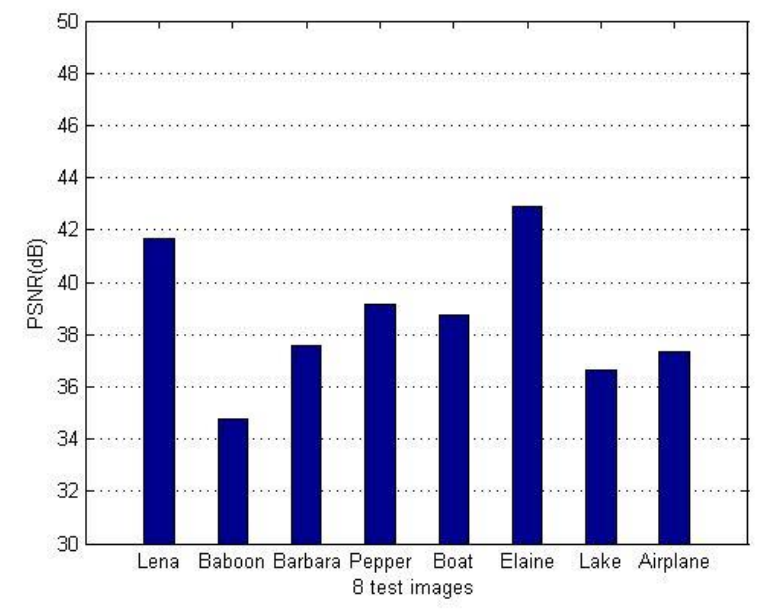

Figure 5. The PSNRs of 8 Watermarked Images 
Generally, most of the watermarking systems measure the robustness of watermarking algorithm with normalized correlation coefficient (NC), but the NC is not universal. It applies only to the judgment of the differences between the binary images, and most of the watermarking algorithms are based on the binary watermark image. The watermark image used in this paper is gray-scale image, and what the extraction algorithm extracts is also the gray-scale image, so we can't use NC to measure the robustness of the watermarking system. Same as the judgment method of invisibility, robustness could be calculated by PSNR also, between the original watermark and extracted watermark. The extracted watermark is more like the original watermark, which means it has a higher robustness.

\subsection{The Embedding Capacity Affects Image Quality}

In order to measure the relationship between the watermark embedding capacity and the image distortion, this algorithm is applied to different test images. The threshold $\mathrm{T}$ is used to adjust the embedding capacity. The smaller $\mathrm{T}$ value brings about higher embedding capacity and more distortion. The higher $\mathrm{T}$ value means lower embedded and better visual effect. Due to the adjustable $\mathrm{T}$ value, we can also adjust the embedding capacity according to the actual needs of users in this algorithm.

The Table 2 represents the experiments under the different $T$ value and the effect of different images. It can be seen that the best image quality is gotten when the $T$ value is biggest. The average PSNR value associated with the highest quality for all the test images exceeds 34dB. The 'Lena' image quality is best when PSNR value is 41.6602. Furthermore, when the $\mathrm{T}$ value is smaller, more secret information can be embedded, and the average PSNR value is still more than $30 \mathrm{~dB}$.

Due to the different smoothness and complexity of the different images themselves, the impact on the embedding capacity is also different. As for a more complex and more texture images, such as "Baboon", we can embed more information with equal $\mathrm{T}$ value than the smooth 'Lena' and 'Elaine'.

Table 2. Embedding Effect in Different $T$ Value

\begin{tabular}{|c|c|c|c|}
\hline Images & The value of $\mathrm{T}$ & Capacity(blocks) & PSNR \\
\hline \multirow{8}{*}{ Lena } & 100 & 11441 & 32.0441 \\
\hline & 300 & 6993 & 33.5583 \\
\hline & 500 & 4889 & 34.9129 \\
\hline & 700 & 3545 & 36.0714 \\
\hline & 900 & 2625 & 37.3159 \\
\hline & 1100 & 2121 & 39.416 \\
\hline & 1300 & 1633 & 40.5137 \\
\hline & 1500 & 1281 & 41.6602 \\
\hline \multirow{8}{*}{ Baboon } & 100 & 27737 & 30.2327 \\
\hline & 300 & 22265 & 31.0339 \\
\hline & 500 & 19041 & 31.5988 \\
\hline & 700 & 16393 & 32.2235 \\
\hline & 900 & 13665 & 32.9581 \\
\hline & 1100 & 11073 & 32.8519 \\
\hline & 1300 & 8913 & 33.7714 \\
\hline & 1500 & 6777 & 34.7863 \\
\hline
\end{tabular}




\begin{tabular}{|c|c|c|c|}
\hline \multirow{4}{*}{ Barbara } & 100 & 19857 & 30.4423 \\
\cline { 2 - 4 } & 300 & 14073 & 31.8473 \\
\cline { 2 - 4 } & 500 & 11097 & 32.8359 \\
\cline { 2 - 4 } & 700 & 8769 & 33.8417 \\
\cline { 2 - 4 } & 900 & 6889 & 34.7432 \\
\cline { 2 - 4 } & 1100 & 5377 & 35.6258 \\
\cline { 2 - 4 } & 1300 & 4193 & 36.5401 \\
\cline { 2 - 4 } & 1500 & 3257 & 37.5993 \\
\hline
\end{tabular}

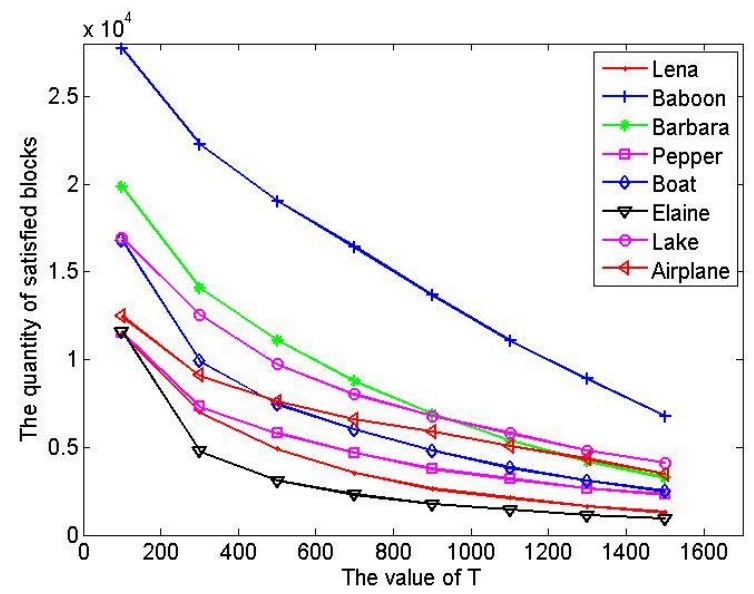

Figure 6. The Quantity of Blocks with Different Value of T

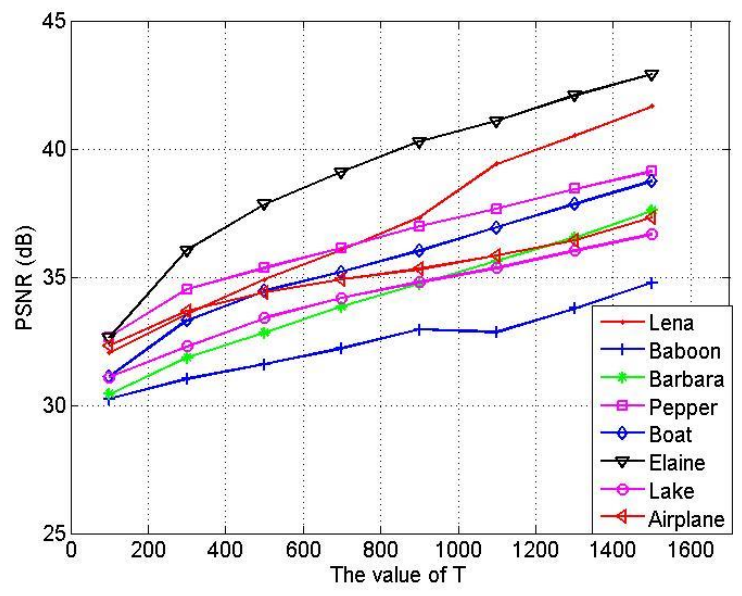

Figure 7. PSNR with Different Value of $\mathrm{T}$

\subsection{Robustness}

The test images with size of $512 \times 512$ as the host image are shown in Figure 4 and 'Lena' with size of $256 \times 256$ as watermark.

When the watermarked image is not attacked, the PSNR value between the watermarked image and the original host image is 34.9129, and the PSNR value between the watermark and the original watermark is 29.18 after being extracted.

Now we will test when faced with the non-geometric attacks and geometric attacks effectiveness of the algorithm respectively. The non-geometric attacks include filtering, JPEG compression. Currently, JPEG is one of the digital image formats most widely used 
on the Internet and digital camera. Quality factor of JPEG is a number from 0 to 100 , which represents the specified degree of compression image. The smaller the value is, the greater the degree of compression is. While, the value is bigger, the smaller the change to the original image is.

\section{Table 3. Experimental Results under Different Attacks of Our Scheme (Measured by PSNR)}

\begin{tabular}{|c|c|c|c|c|}
\hline Test images & $\begin{array}{c}\text { Median } \\
\text { filter }\end{array}$ & $\begin{array}{c}\text { JPEG } \\
\mathrm{QF}=80\end{array}$ & $\begin{array}{c}\text { JPEG } \\
\mathrm{QF}=50\end{array}$ & $\begin{array}{c}\text { JPEG } \\
\mathrm{QF}=30\end{array}$ \\
\hline Lena & 24.32 & 24.1 & 22.91 & 20.67 \\
\hline Baboon & 26.83 & 26.34 & 24.71 & 22.92 \\
\hline Barbara & 23.15 & 22.98 & 21.64 & 20.51 \\
\hline
\end{tabular}

Our experiments of geometric attacks include cropping and additive noising attack. For additive noising attack, we tested Gaussian noise attack and salt \& pepper noise. For cropping attack operation, it is to cut off a quarter of the image.

The experimental results after the attacks are shown in the Table 4.

Table 4. Experimental Results under Different Attacks

\begin{tabular}{|c|c|c|c|}
\hline $\begin{array}{c}\text { Test } \\
\text { images }\end{array}$ & $\begin{array}{c}\text { Cropping } \\
1 / 4\end{array}$ & $\begin{array}{c}\text { salt \& pepper } \\
\text { noise }\end{array}$ & $\begin{array}{c}\text { Gaussian } \\
\text { noise }\end{array}$ \\
\hline Lena & 28.67 & 29.11 & 28.53 \\
\hline Baboon & 30.02 & 31.54 & 29.8 \\
\hline Barbara & 28.17 & 28.91 & 27.16 \\
\hline
\end{tabular}

We compared our method with other methods, such as Run's [15] and Wu's [16]. Due to the fewer articles using gray-level watermark embedding, we just take two articles based on the binary watermark image to make a simple comparison. The host image they used and we used are of the same size, which are $512 \times 512$ gray images. The size of embedded watermark image of X.T. Wu is $64 \times 64$. Among them, the watermark image of Ray-Shine Run is even smaller. As to the robustness of watermarking system, because what the measured values the binary image uses is $\mathrm{NC}$ value, the gray watermark image we used takes use of PSNR value. Both of them can't compare directly. And by comparison with the visual effect of the experimental results, it can be seen that these algorithms has good robustness in the face of both geometric attacks and non-geometric attacks.

In terms of invisibility, it can be seen that at the case of the similar size of embedded watermark, the PSNR value our algorithm obtained and X.T. Wu's and Ray-Shine Run's are similar. And this algorithm used the gray-level watermark, compared to the same size of the binary watermark, it contains more image details, the amount of information and the number of bits, which is equivalent to embedding more capacity of information. Therefore, this method has good invisibility.

\section{Conclusions}

This paper presents a new gray scale watermarking algorithm of the wavelet domain based on watermark and image preprocessing. The watermark preprocessing has greatly reduced the embedded watermark bits, or has greatly improved the capacity of embedded 
watermark with the same visual effect. The embedding process is based on human visual system's characteristic. Threshold $\mathrm{T}$ is used to determine the quantity of embedded blocks, and control the capacity of embedded secret information. Experiments show that the algorithm has a good robustness for general attacks.

\section{References}

[1] Daniel Lerch-Hostalot, David Megías, "LSB matching steganalysis based on patterns of pixel differences and random embedding”, Computers \& Security, Vol.32, (2013) pp.192-206.

[2] J. Kong and W. Wang, "Joint spatial and frequency domains watermarking algorithm based on wavelet packets transform", The 18th Australian Joint Conference on Artificial Intelligence, (2005)

[3] B. Abdullah and I. Rosziati, "A New Digital Watermarking Algorithm Using Combination of Least Significant Bit (LSB) and Inverse Bit”, JOURNAL OF COMPUTING, Vol.3, No.4, (2011) pp.1-8.

[4] C.F. Lee and H.S. Chen, "Adjustable prediction-based reversible data hiding", Digital Signal Processing, Vol.22, (2012) pp.941-953.

[5] Tzu-Chuen Lu, Chun-Ya Tseng, Jhih-Huei Wu, "Dual imaging-based reversible hiding technique using LSB matching", Signal Processing, Vol.108, (2015) pp.77-89.

[6] Jiu-fen Liu, Yu-guo Tian, Tao Han, Chun-fang Yang, Wen-bin Liu, "LSB steganographic payload location for JPEG-decompressed images”, Digital Signal Processing, Vol.38, (2015) pp.66-76.

[7] Das C, Panigrahi D, Sharma VK, Mahapatra K.K, "A novel blind robust image watermarking in DCT domain using inter-block coefficient correlation" AEU - International Journal of Electronics and Communications, Vol.68, No.3, (2014) pp.244-253.

[8] Y. Yu and M. Lei, "A Novel Robust Zero-Watermarking Scheme Based on Discrete Wavelet Transform", Journal of Multimedia, Vol.7, No.4, (2012).

[9] W. Lin, Y. Wang, S. Horng and T. Kao, "A blind watermarking method using maximum wavelet coefficient quantization", Expert Systems with Applications, Vol.23, (2009) pp.11509-11516.

[10] A. Santa and A. Guido, "A new approach to pre-processing digital image for wavelet-based watermark", Journal of Computational and Applied Mathematics, Vol. 21, (2008) pp.274-283. Yih-Kai Lin, "A data hiding scheme based upon DCT coefficient modification", Computer Standards \& Interfaces, Vol.36, No.5, (2014) pp. 855-862.

[11] W.C. Hu and W.H. Chen, "Robust image watermarking based on discrete wavelet transform-discrete cosine transform-singular value decomposition", J. Electron. Imaging, Vol.21, (2012).

[12] Suk-Hwan Lee, "DWT based coding DNA watermarking for DNA copyright protection", Information Sciences, Vol.273, No.20, (2014) pp.263-286.

[13] M.S. Hsieh, D.C. Tseng, and Y.H. Huang, "Hiding digital watermarks using multiresolution wavelet transform", IEEE Transactions on Industrial Electronics, Vol.48, (2001) pp.875-882.

[14] W. Lin, S. Horng, T. Kao andP. Fan, "An efficient watermarking method based on significant difference of wavelet coefficient quantization”, IEEE Transactions on Multimedia, Vol.10, (2008) pp.746-757.

[15] Ray-Shine, Run and Shi-Jinn, Horng, "An efficient wavelet-tree-based watermarking method", Expert Systems with Applications, Vol. 38, (2011) pp. 14357-14366.

[16] X.T. Wu, W. Sun, "Robust copyright protection scheme for digital images using overlapping DCT and SVD”, Applied Soft Computing, Vol.13, (2013) pp.1170-1182.

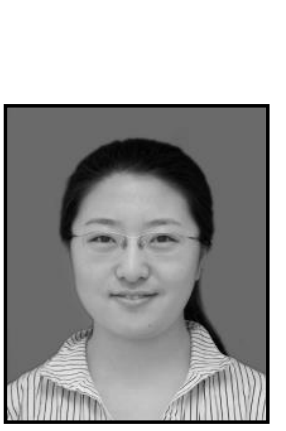

\section{Authors}

Jialing Han, she is a Ph.D. student in the college of communication engineering at Jilin University in Changchun, China. Her research interests are in the areas of information security, information hiding and digital watermark. 


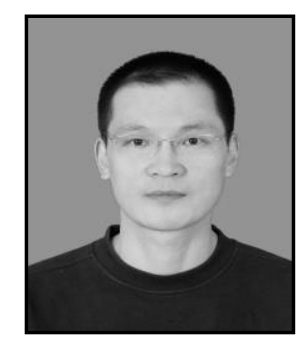

Xiaohui Zhao, he is a professor in the college of communication engineering at Jilin University. He received his Ph.D degree in 1993. His research interests include the theory of digital signal processing, speech signal processing. 